\title{
Design, synthesis and RAFT polymerisation of a quinoline-based monomer \\ for use in electrospun composite microfibres
}

\author{
Anna Isakova, ${ }^{a}$ Olga Efremova,${ }^{b}$ Nikki Pullan, ${ }^{a}$ Larry Lüer, ${ }^{c}$ Paul D. Topham ${ }^{a}$ \\ a Chemical Engineering and Applied Chemistry, Aston University, Aston Triangle, \\ Birmingham, B4 7ET, UK; \\ ${ }^{\mathrm{b}}$ Department of Chemistry, University of Hull, Hull, HU6 7RX, UK; \\ c IMDEA Nanociencia, C/ Faraday, 9, Cantoblanco, 28049, Madrid, Spain. \\ * Corresponding author: p.d.topham@aston.ac.uk.
}

Metal-binding polymer fibres have attracted major attention for diverse applications in membranes for metal sequestration from waste waters, non-woven wound dressings, matrices for photocatalysis, and many more. This paper reports the design and synthesis of an 8hydroxyquinoline-based zinc-binding styrenic monomer, QuiBoc. Its subsequent polymerisation by reversible addition-fragmentation chain transfer (RAFT) yielded welldefined polymers, PQuiBoc, of controllable molar masses (6 and $12 \mathrm{~kg} / \mathrm{mol}$ ) with low dispersities $\left(\nexists, M_{\mathrm{w}} / M_{\mathrm{n}}<1.3\right)$. Protected (PQuiBoc) and deprotected (PQuiOH) derivatives of the polymer exhibited high zinc-binding capacity, as determined by semi-quantitative SEM/EDXA analyses, allowing the electrospinning of microfibres from a PQuiBoc/polystyrene (PS) blend without the need for removal of the protecting group. Simple "dip-coating" of the fibrous mats into $\mathrm{ZnO}$ suspensions showed that PQuiBoc/PS microfibres with only $20 \%$ PQuiBoc content had almost three-fold higher loadings of $\mathrm{ZnO}(29 \%)$ in comparison to neat PS microfibres (11\%).

\section{Introduction}

Organic-inorganic composite polymer materials continue to draw large attention due to their attractive properties, combining the processability and physical properties of an organic polymer component with the functionality of an inorganic moiety. ${ }^{1}$ One of the most extensively 
employed techniques for the preparation of the composite materials with high surface area and loading of inorganic components is electrospinning, an electro-hydrodynamic process that allows the fabrication of nano- and microfibres with a wide range of secondary structures, compositions and applications: from biological scaffolds to water purification. ${ }^{2}$ The main advantage of fibres is their high surface area-to-volume ratio and outstanding mechanical properties. $^{3}$

Polystyrene (PS) fibres have been of major interest as a readily available material in a range of molar masses that exhibits excellent physical properties, such as high tensile and flexural stress with high long-term stability. ${ }^{4,5}$ Moreover, good control over the size, size distribution, wettability ${ }^{6}$ and the morphology of the fibres has been achieved, including formation of pores, ${ }^{7,8}$ wrinkles and folds. ${ }^{9}$ A good understanding of the structure-property relationship of the electrospun PS fibres and their relatively low cost have led to PS being the first choice material for producing fibres for diverse applications, including gas and chemical sensors, ${ }^{9}$ fluorescent $\mathrm{pH}$-sensors,${ }^{10}$ oil clean-up sorbents, ${ }^{1-14}$ ion-exchange membranes,${ }^{15}$ antibacterial wound dressings, ${ }^{16}$ biological scaffolds ${ }^{17}$ among others. Fibres of more sophisticated morphologies can be obtained by electrospinning PS-containing block copolymers of various polymer architectures. ${ }^{10,18-20}$ These block copolymer fibres exhibit advantageous properties, such as highly enhanced hydrophobicity, ${ }^{20}$ elastomeric properties and liquid-crystalline behavior. ${ }^{21}$ Furthermore, simple fabrication and solvent compatibility with various materials has allowed subsequent layer-by-layer functionalisation of the PS fibres with polyelectrolytes, deoxyribonucleic acids and composite polymer/nanoparticle layers to obtain hollow composite fibres. $^{22}$

Another advantage of electrospun PS fibres is that the polymer solutions can be loaded with various additives and components. Additives such as cellulose, sisal or single-walled carbon nanotubes have been used to improve the mechanical properties of the fibres. ${ }^{23-25}$ Furthermore, PS nanofibres are often used as scaffolds, or matrices, for the incorporation of inorganic 
nanoparticles, such as gold, ${ }^{26}$ silica, ${ }^{27} \mathrm{TiO}_{\mathrm{x}}$ and $\mathrm{ZnO}^{28}$ or serving as templates for the fabrication of such nanoparticles. ${ }^{16}$ For example, incorporation of $\mathrm{Fe}_{2} \mathrm{O}_{3}$ in nanofibres for the removal of oil from water, whilst allowing facile removal of the nanofibres after the sorption process,${ }^{29}$ or loading a PS fibre matrix with gold nanoparticles in for the colorimetric detection of oestrogenic compounds in dairy products. ${ }^{30}$

However, the post-spinning modification of PS nanofibres with a high loading of nanoparticles has so far been inaccessible because of the limited interactions between PS and the nanoparticles, although for many applications the distribution of the particles on the fibre surface is important for the functionality, e.g. in wound dressings or photocatalysis. ${ }^{31,32}$

To this end, a novel tert-butyloxycarbonyl (Boc) protected 8-hydroxyquinoline monomer has been designed and polymerised by reversible addition-fragmentation chain transfer (RAFT) polymerisation for subsequent blending with PS to create microfibres with high metal-binding capability. The design of this monomer has been inspired by previous reports of the metalbinding property of the 8-hydroxyquinoline moiety in various applications, such as emitters or electron-transporting layers (as a complex with aluminium or zinc, $\mathrm{Alq}_{3}$ or $\mathrm{Znq}_{2}$ ) in organic light emitting devices (OLEDs), ${ }^{33-35}$ photoconductors, ${ }^{36}$ or even as potential antineurodegenerative or antibiotic drugs (such as clioquinol). ${ }^{37}$ Our approach herein exploits the use of low molar mass PQuiBoc/PS/ZnO microfibres which are highly loaded with $\mathrm{ZnO}$ post-spinning by simply "dip-coating" the fibre mats in $\mathrm{ZnO}$ suspensions. Moreover, to decrease the number of processing steps, and therefore fabrication costs, the PQuiBoc polymer is introduced into the blend without deprotection, allowing high $\mathrm{ZnO}$ surface loadings on the fibre even at low PQuiBoc loadings (2:8 PQuiBoc:PS). 


\section{Experimental}

\section{Materials}

5-Bromo-8-hydroxyquinoline (> 96.0\%, TCI Europe N.V.), 5-chloro-8-hydroxyquinoline (95\%, Alfa Aesar), 2-dicyclohexylphosphino-2',4',6'-triisopropylbiphenyl (Xphos, 98+\%, Alfa Aesar), bis(acetonitrile)dichloropalladium(II) (Pd 40.5\%, Alfa Aesar), tetrakis(triphenylphosphine)palladium(0) (99.8\% metals basis, Pd 9\% min, Alfa Aesar), ditert-butyl dicarbonate (ReagentPlus ${ }^{\circledR}, \geq 99 \%$, Sigma Aldrich), copper iodide (99.999\% trace metals basis, Sigma Aldrich), $N$-ethyldiisopropylamine (DIPEA, 99\%, Sigma Aldrich), 4(dimethylamino)pyridine (DMAP, ReagentPlus ${ }^{\circledR}, \quad \geq \quad 99 \%, \quad$ Sigma Aldrich), ethynyltrimethylsilane (98\%, Alfa Aesar), sodium azide (ReagentPlus ${ }^{\circledR}, \geq 99.5 \%$, Sigma Aldrich), potassium fluoride (anhydrous powder, $\geq 99.99 \%$ trace metals basis, Sigma Aldrich), zinc oxide nanoparticles 40-100 nm NanoArc $®$ ZN-2605 (Alfa Aesar), acetone, propan-2-ol (IPA), methanol, diethyl ether, dichloromethane (all Fisher Scientific, Laboratory grade) were used as received. 4-Vinylbenzyl chloride (VBC, 90\%, Sigma Aldrich) was extracted with 10\% aqueous $\mathrm{NaOH}$ solution to remove the inhibitor. 2,2'-Azobis(isobutyronitrile) (AIBN, Fisher Scientific) was recrystallised from methanol and dried in vacuo before use. 2(Dodecylthiocarbonothioylthio)-2-methylpropionic acid (DDMAT) was synthesised according to Lai et $a l .{ }^{38}$ and recrystallised from hexane before use. 4-Vinylbenzyl azide was synthesised according to $\mathrm{Li}$ et al. ${ }^{39}$ Anhydrous tetrahydrofuran (THF, $\geq 99.9 \%$, inhibitor free, Sigma Aldrich) and acetonitrile (anhydrous, 99.8\%, Sigma Aldrich) were purged with nitrogen before use. Polystyrene (PS, $M_{\mathrm{n}} \sim 5 \mathrm{~kg} / \mathrm{mol}$ ) was synthesised according to a previous report. ${ }^{40}$

\section{Characterisation methods}

${ }^{1} \mathrm{H}$ and ${ }^{13} \mathrm{C}$ NMR spectra were recorded using a Bruker NMR spectrometer (300 MHz). All chemical shifts are reported in ppm $(\delta)$ and referenced to the chemical shifts of the residual solvent resonances. 
Fourier transform infrared (FTIR) spectra were obtained using $\mathrm{KBr}$ discs on a Perkin Elmer Spectrum One spectrometer over the range $4000-500 \mathrm{~cm}^{-1}$ for 16 scans with a resolution of $4 \mathrm{~cm}^{-1}$.

Number-average molar mass $\left(M_{\mathrm{n}}\right)$ and dispersity $\left(M_{\mathrm{w}} / M_{\mathrm{n}}, Ð\right)$ were measured using gel permeation chromatography (GPC) (flow rate $1 \mathrm{ml} / \mathrm{min}$ ) through three PL gel $5 \mathrm{~mm} 300 \times$ $7.5 \mathrm{~mm}$ mixed-C columns using a degassed THF eluent system containing $2 \%(\mathrm{v} / \mathrm{v})$ triethylamine. The system, operating at $40{ }^{\circ} \mathrm{C}$, was calibrated with narrow PS standards $\left(M_{\mathrm{p}}\right.$ range $=162$ to $6035000 \mathrm{~g} / \mathrm{mol}$ ). All data were analysed using PL Cirrus software (version 2.0) supplied by Agilent Technologies (previously Polymer Laboratories).

Ultraviolet and visible (UV-Vis) absorbance spectra were obtained using a Perkin Elmer Lambda 35 system in the wavelength range $300-800 \mathrm{~nm}$, using THF as solvent at $0.01 \mathrm{mg} / \mathrm{ml}$. The photoluminescence spectra were obtained on a Hitachi FL-2500 spectrophotometer with an excitation wavelength at the maximum absorbance.

The glass transition temperature, $T_{\mathrm{g}}$, and deprotection temperature of the polymer $\left(T_{\mathrm{dep}}\right)$ were measured using differential scanning calorimetry (DSC) equipment DSC 1 STARe (Mettler Toledo) at a heating rate of $10{ }^{\circ} \mathrm{C} / \mathrm{min}$ under nitrogen atmosphere with flow rate of $60 \mathrm{ml} / \mathrm{min}$. Typically, one analysis included a heating step from 0 to $300^{\circ} \mathrm{C}$, a cooling step to $0{ }^{\circ} \mathrm{C}$, and a second heating run from 0 to $200{ }^{\circ} \mathrm{C}$.

\section{Synthesis of the monomer precursor 8-tert-butoxycarbonyloxy-5-ethynylquinoline (3)}

An oven-dried round-bottom flask was backfilled twice with nitrogen atmosphere and charged with $3.0 \mathrm{~g}(9.25 \mathrm{mmol})$ of the protected 5-bromo-8-hydroxyquinoline, $500 \mathrm{mg}$ of $\mathrm{Pd}\left(\mathrm{PPh}_{3}\right)_{4}$ and $88 \mathrm{mg}(0.46 \mathrm{mmol})$ of $\mathrm{CuI}$ and $350 \mathrm{ml}$ of dry THF. The solution was purged with nitrogen for $15 \mathrm{~min}$. The flask was equipped with a magnetic follower and sealed with a rubber septum. DIPEA $(30 \mathrm{ml})$ and trimethylsilylacetylene $(6.54 \mathrm{ml}, 46.27 \mathrm{mmol})$ were added through the rubber septum and the reaction mixture stirred at $60{ }^{\circ} \mathrm{C}$ for $24 \mathrm{~h}$. The mixture was filtered 
through celite to remove the copper salts, using diethyl ether as eluent. The filtrate was concentrated and the residue redissolved in methanol. Resulting mixture was stirred with $800 \mathrm{mg}$ of potassium fluoride at room temperature for $5 \mathrm{~h}$. To the mixture filtered through a filter paper, $40 \mathrm{ml}$ of water added and methanol was evaporated at room temperature. The residue was extracted with dichloromethane $(2 \times 300 \mathrm{ml})$. Organic layer was separated, dried over magnesium sulfate and concentrated under vacuum. Final product was purified by column chromatography (15\% ethyl acetate in hexane) to yield $2.26 \mathrm{~g}$ of yellow powder (yield $91 \%$ ). ${ }^{1} \mathrm{H}$ NMR $\left(\mathrm{CDCl}_{3}, \delta \mathrm{ppm}\right): 1.59$ (s, $\left.9 \mathrm{H}\right), 3.47(\mathrm{~s}, 1 \mathrm{H}), 7.48(\mathrm{~d}, J=7.9 \mathrm{~Hz}, 1 \mathrm{H}), 7.52(\mathrm{dd}, J=$ $4.2,8.5 \mathrm{~Hz}, 1 \mathrm{H}), 7.76(\mathrm{~d}, J=7.9 \mathrm{~Hz}, 1 \mathrm{H}), 8.63(\mathrm{dd}, J=1.7,8.5 \mathrm{~Hz}, 1 \mathrm{H}), 8.96 \mathrm{ppm}(\mathrm{dd}, J=$ $1.7,4.2 \mathrm{~Hz}, 1 \mathrm{H})$.

Synthesis of the monomer, tert-butyl-\{5-[2-(4-vinylbenzyl)-2H-1,2,3-triazol-4-yl]quinolin-8yly) carbonate QuiBoc (5)

A solution of $\mathbf{3}(1.2 \mathrm{~g}, 4.45 \mathrm{mmol})$, 4-vinylbenzyl azide (4, $0.71 \mathrm{~g}, 4.46 \mathrm{mmol})$ and $5 \mathrm{mg} \mathrm{CuI}$ in dry THF was charged into a round-bottom flask and purged with nitrogen and stirred for 15 minutes. After purging, the solution was heated to $40{ }^{\circ} \mathrm{C}$ and left stirring overnight. The solvent was evaporated and the solid redissolved in DCM and washed with water to remove the copper catalyst. The organic phase was subsequently dried over magnesium sulfate. The solvent was evaporated under vacuum and the residue purified using column chromatography on silica gel (ethyl acetate:hexane, 1:1) to provide the product (1.83 g, yield 96\%) as a white powder. M.p. 136-138 ${ }^{\circ} \mathrm{C} .{ }^{1} \mathrm{H}$ NMR $\left(\mathrm{CDCl}_{3}, \delta \mathrm{ppm}\right): 1.62(\mathrm{~s}, 9 \mathrm{H}), 5.31(\mathrm{~d}, J=10.9 \mathrm{~Hz}, 1 \mathrm{H}), 5.67(\mathrm{~s}, 2 \mathrm{H})$, $5.75(\mathrm{~d}, J=17.5 \mathrm{~Hz}, 1 \mathrm{H}), 6.69(\mathrm{dd}, J=17.5,10.9 \mathrm{~Hz}, 1 \mathrm{H}), 7.34(\mathrm{~d}, J=8.2 \mathrm{~Hz}, 2 \mathrm{H}), 7.46-7.49$ $(\mathrm{d}, J=8.2 \mathrm{~Hz}, 2 \mathrm{H}), 7.45-7.51(\mathrm{dd}, J=4.2,8.2 \mathrm{~Hz}, 1 \mathrm{H}), 7.53(\mathrm{~d}, J=7.85 \mathrm{~Hz}, 1 \mathrm{H}), 7.65(\mathrm{~d}, J=$ $7.85 \mathrm{~Hz}, 1 \mathrm{H}), 7.72(\mathrm{~s}, 1 \mathrm{H}), 8.93(\mathrm{dd}, J=8.2,1.6,1 \mathrm{H}), 8.98(\mathrm{dd}, J=1.6,4.21 \mathrm{H}) .{ }^{13} \mathrm{C}$ PENDANT NMR $\left(\mathrm{CDCl}_{3}, \delta \mathrm{ppm}\right): 25.75\left(\mathrm{CH}_{3}\right), 54.33\left(\mathrm{CH}_{2}\right), 83.90\left(\mathrm{C}^{*}\right), 115.28\left(\mathrm{CH}_{2}\right) 122.18$ (C*), $122.3(\mathrm{CH}), 127.02(\mathrm{CH}), 127.47(\mathrm{CH}), 133.61\left(\mathrm{C}^{*}\right), 134.77(\mathrm{CH}), 138.40\left(\mathrm{C}^{*}\right), 150.62$ $(\mathrm{C}=\mathrm{O})$. ESI-MS [M+]: found 429.1909, calculated: 429.1927. Elemental analysis calcd. (\%) for 
$\mathrm{C}_{25} \mathrm{H}_{24} \mathrm{~N}_{4} \mathrm{O}_{3}: \mathrm{C} 70.08 \%, \mathrm{H} 5.65 \%, \mathrm{~N} 13.07 \%, \mathrm{O} 11.20 \%$; found $\mathrm{C} 70.02 \%$; $\mathrm{H} 5.63 \%, \mathrm{~N} 13.22 \%$, O $11.13 \%$.

\section{RAFT polymerisation of QuiBoc (5)}

The following procedure describes the polymerisation of QuiBoc in THF at $60{ }^{\circ} \mathrm{C}$ with $[\mathrm{AIBN}]_{0} /[\mathrm{DDMAT}]_{0} /[\mathrm{QuiBoc}]_{0}=1 / 1 / 20$ (i.e. a target degree of polymerisation, $D_{\mathrm{p}}$, of 20 ); this is representative of all QuiBoc polymerisations undertaken in this work (Scheme 2). A $25 \mathrm{ml}$ polymerisation tube equipped with a magnetic follower was charged with a mixture of monomer (0.4 g, $0.93 \mathrm{mmol})$, AIBN (7.7 mg, $0.047 \mathrm{mmol})$, DDMAT (17 mg, $0.047 \mathrm{mmol})$ and THF (4 ml). The flask was sealed with a rubber septum and the solution was stirred and purged with nitrogen for 15 minutes. Following which, the flask was placed in an oil bath at $60{ }^{\circ} \mathrm{C}$. After $36 \mathrm{~h}$ polymerisation was cooled rapidly to $0{ }^{\circ} \mathrm{C}$ to quench the polymerisation. $10 \mathrm{ml} \mathrm{THF}$ was added and the resulting solution was precipitated in $100 \mathrm{ml}$ of cold diethyl ether. The PQuiBoc precipitate was collected by filtration, washed with cold diethyl ether multiple times and dried in vacuo.

\section{Deprotection of PQuiBoc (6) to obtain PQuiOH (7)}

PQuiBoc polymer $(20 \mathrm{mg}$ ) was dissolved in $5 \mathrm{ml}$ of DCM before 2 drops of piperidine were added to the solution, with stirring. After 5 min the colour of the polymer solution transformed from colourless to yellow and later precipitated as pale yellow flakes. Filtration afforded $17 \mathrm{mg}$ of PQuiOH product (yield 98\%).

\section{Electrospinning}

In a typical electrospinning procedure, a polymer solution (33 wt $\%$ ) was prepared in chloroform by dissolving $120 \mathrm{mg}$ of PS ( $5 \mathrm{~kg} / \mathrm{mol})$ and $30 \mathrm{mg}$ of PQuiBoc 14 ( $\sim 6 \mathrm{~kg} / \mathrm{mol})$, in a 2:8 ratio. The solution was sonicated for 10 minutes until complete dissolution of the solids was achieved. DC high-voltage generator (Genvolt lab unit 73030) was applied to produce voltages ranging from 0 to $25 \mathrm{kV}$. Polymer solutions were charged into a $1 \mathrm{ml}$ syringe and fed into the needle capillary tip (inner diameter $0.3 \mathrm{~mm}$ ) by a syringe pump at a speed of $3.5 \mathrm{ml} / \mathrm{h}$. 
A collecting sheet of aluminium foil, connected to the ground, was placed $20 \mathrm{~cm}$ from the needle tip. Voltage was supplied to the needle of the tip at a constant value of $20 \mathrm{kV}$.

\section{Fibre characterisation}

Surface morphology and zinc binding performance were studied using scanning electron microscopy with energy dispersive X-ray analysis (SEM/EDXA) on a Zeiss EVO60 microscope, fitted with Oxford Instruments Inca System350 Energy Dispersive X-ray Spectrometer $(\mathrm{EDX})$ at a beam intensity of $20 \mathrm{kV}$ and a probe current of $130 \mathrm{pA}$. Before imaging the polymer fibres, mats were coated with a layer of carbon of the (10 $\mathrm{nm}$ approximate thickness). The polymer fibre mat was imaged pristine or after soaking in a methanol suspension of zinc oxide $(\mathrm{ZnO})$ nanoparticles and subsequent multiple washing with methanol and sonication to remove any non-bound zinc oxide.

\section{Results and discussion}

\section{Monomer synthesis}

The monomer was specifically designed to satisfy two main criteria: (i) possess an 8hydroxyquinoline moiety for strong metal chelation and (ii) contain a styrenic group for simple polymerisation and to allow compatibility with the PS component of the fibres. Synthesis of the monomer (QuiBoc) was achieved in a small number of high yielding steps (>90\%, Scheme $1)$.<smiles>Oc1ccc(Br)c2ncccc12</smiles>

1

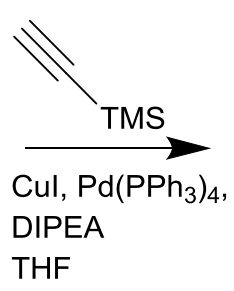

2<smiles>C#Cc1ccc(OC(C)(C)C)c2ncccc12</smiles>

3

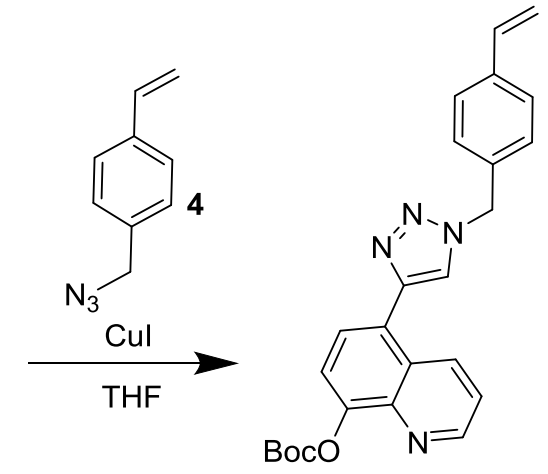

QuiBoc 5

Scheme 1. Synthetic route toward the QuiBoc monomer. 
The starting material, aryl bromide, was first protected with a tert-butyloxycarbonyl (Boc) group. This group provides several advantages, since (i) it is stable under the mild basic conditions of Sonogashira coupling and (ii) it can be easily removed (chemically or by thermal treatment).

Synthesis of 8-tert-butoxycarbonyloxy-5-ethynylquinoline (3) was carried out by Sonogashira coupling. This is an efficient way to form a C-C bond between the aryl bromide 2 (or a corresponding iodide) and trimethylsilylacetylene, whereas aryl chlorides are not converted under these conditions and normally require a Buchwald ligand and copper salt. ${ }^{41}$ However, in this work it was found that the coupling of aryl chloride can be carried out using 2dicyclohexylphosphino-2',4',6'-triisopropylbiphenyl (XPhos) ligand in combination with an acetonitrile palladium complex, both commercially available, without the addition of copper salt. Furthermore, caesium carbonate, usually employed in aryl chloride cross-coupling as a base, cannot be used in this reaction as it would cause the protecting group to be removed. Thus, $N$-ethyldiisopropylamine (DIPEA) has been proved to be suitable here. Other ligands, such as (2-biphenyl)di-tert-butylphosphine (Johnphos) or 2-dicyclohexylphosphino-2',6'diisopropoxybiphenyl (Ruphos), were not found to be efficient.

4-Vinylbenzyl azide, precursor 4, was synthesised from commercially available 4-vinylbenzyl chloride. The most critical step of the synthesis was the 'click reaction' of 8-tertbutoxycarbonyloxy-5-ethynylquinoline (3) with 4-vinylbenzyl azide (4) which had to be carried out at room temperature to prevent spontaneous polymerisation of $\mathbf{4}$ and other side reactions. It was observed that the reaction could proceed without a copper catalyst, but using a copper (I) salt increased the rate and conversion (up to quantitative).

The efficiency of the 'click reaction' was confirmed by ${ }^{1} \mathrm{H}$ NMR and FTIR (see ESI, Figure S1) where the signal corresponding to the terminal alkynyl proton at $3.5 \mathrm{ppm}$ (labelled as $\mathrm{H}_{\mathrm{a}}$ in Figure 1) of precursor 3 was significantly shifted to 7.73 ppm (Figure 1b) due to the different environment of this proton, transformed to be a part of a 1,2,3-triazole ring (Figure 1). 
(a)
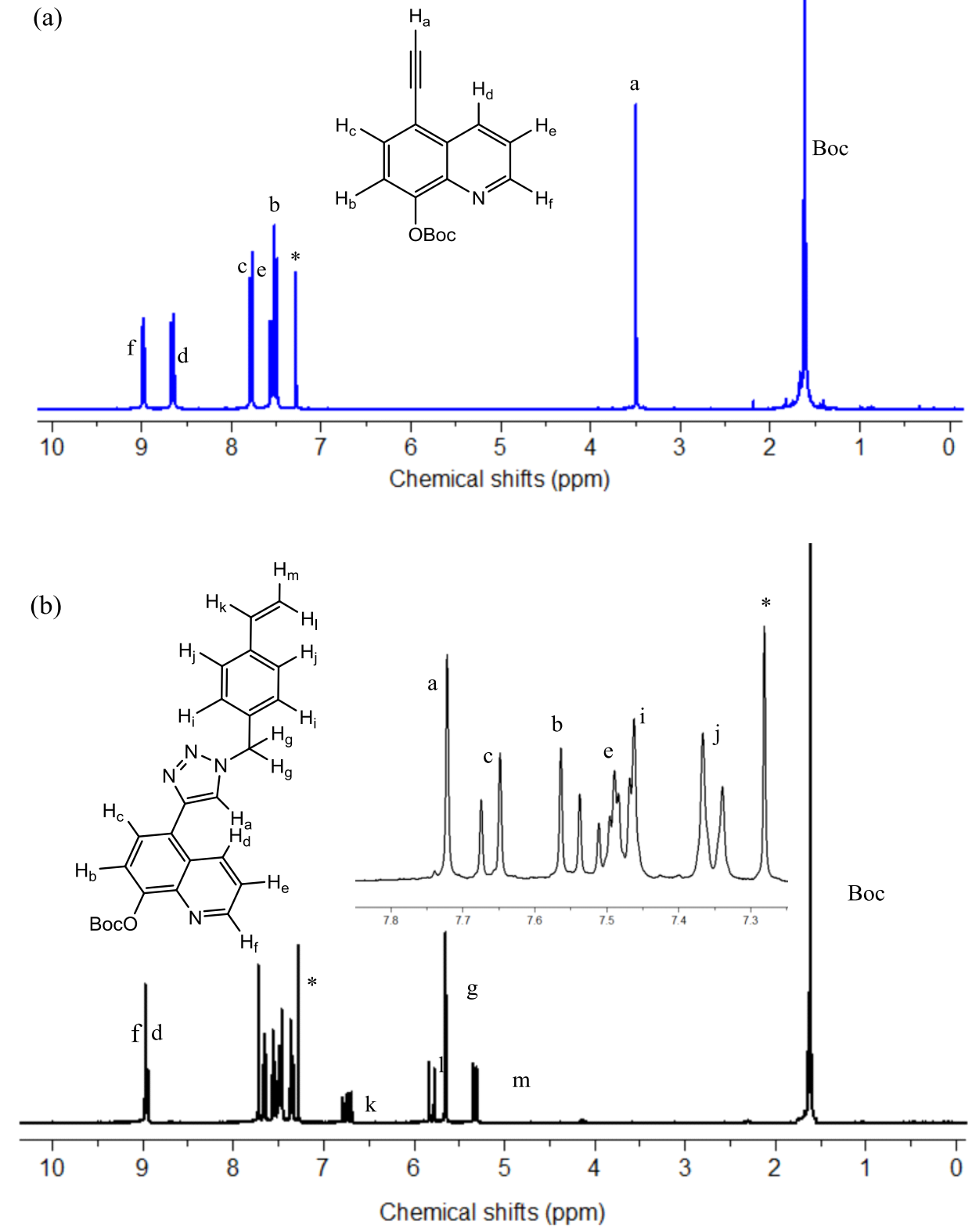

Figure 1. ${ }^{1} \mathrm{H}$ NMR spectra of precursor 3 (a) and QuiBoc monomer 5 (b); residual solvent peaks are labelled with asterisks.

\section{RAFT polymerisation and subsequent deprotection}

To obtain well-defined polymers of QuiBoc, reversible addition-fragmentation chain transfer (RAFT) polymerisation was employed (Scheme 2) owing to its good control over polymer chain length and architecture for various monomers. ${ }^{42-45}$ The use of a chain transfer agent (CTA) allows further modification of the resulting polymers, including synthesis of block copolymers $^{46,47}$ or derivatisation of the polymer with single molecules. ${ }^{48}$ Furthermore, 
appropriate CTAs allow polymerisation from a solid substrate or anchoring the final polymer to nanoparticles. ${ }^{49,50}$

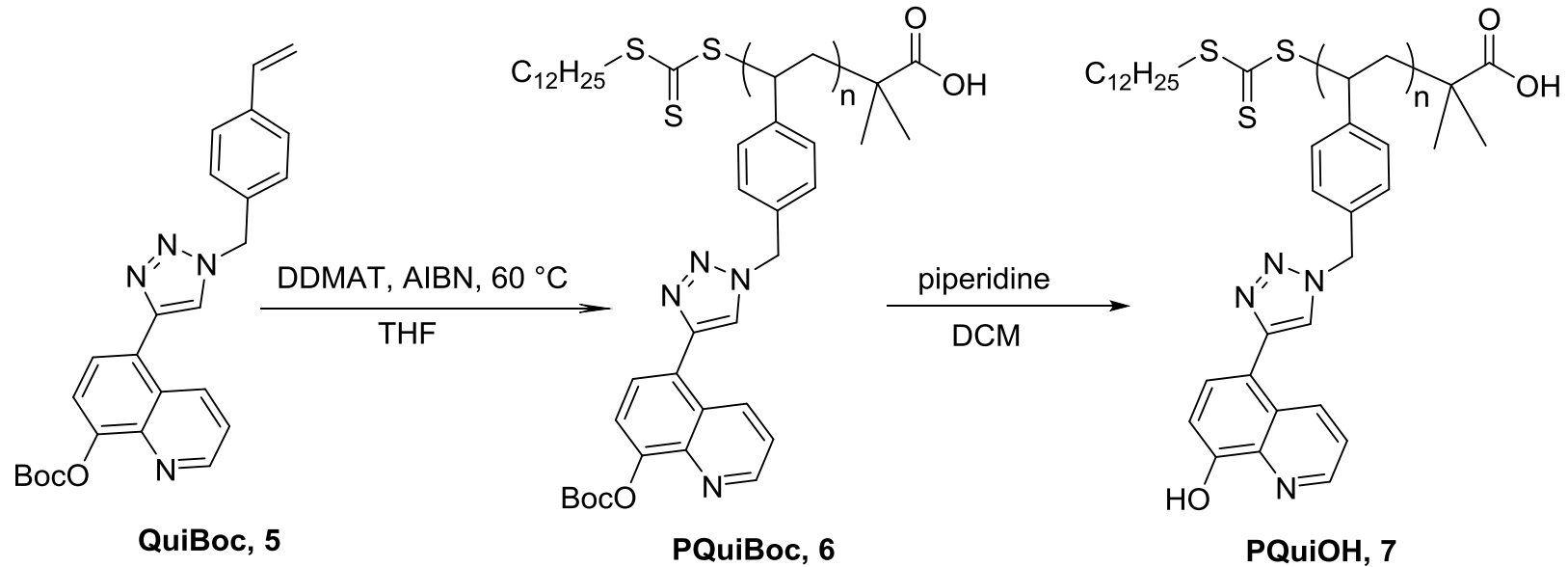

Scheme 2. RAFT polymerisation of QuiBoc monomer 5 and subsequent deprotection to obtain PQuiOH (7).

Based on previous reports, 2-(dodecylthiocarbonothioylthio)-2-methylpropionic acid (DDMAT) was chosen as the CTA, owing to its efficiency in the polymerisation of other styrenic monomers. ${ }^{51,52}$ The initiator to CTA ratio was set to $1: 1\left([\mathrm{AIBN}]_{0} /[\mathrm{DDMAT}]_{0}=1.0\right)$ and the solvent, as well as the monomer concentration, was chosen based on solubility studies of the monomer in various organic solvents. Toluene and anisole were found to be unsuitable for the QuiBoc monomer, whereas THF allowed a solvent to solids ratio of 10:1 v/w (i.e. $10 \mathrm{ml}$ of THF per $1 \mathrm{~g}$ of QuiBoc) to be attained. Polymerisations of QuiBoc with target $D_{\mathrm{p}}$ of 20 and 40 were undertaken; their results are summarised in Table 1. 
Table 1. Summary of monomer conversion and molar mass data for the homopolymerisation of QuiBoc using AIBN and DDMAT in THF at $60^{\circ} \mathrm{C}$.

\begin{tabular}{|c|c|c|c|c|c|c|}
\hline $\begin{array}{c}\text { Target } \\
\qquad D_{\mathrm{p}}\end{array}$ & Target $M_{\mathbf{n}}$ & Time & Conversion $^{\mathrm{a}}$ & $D_{\mathbf{p}}^{\mathrm{b}}$ & $\begin{array}{c}M_{\mathrm{n}^{\mathrm{b}}} \\
(\mathrm{kg} / \mathrm{mol})\end{array}$ & $\begin{array}{c}M_{\mathrm{w}} / M_{\mathrm{n}}, \\
\boldsymbol{Ð}\end{array}$ \\
\hline 20 & 8.9 & 36 & 91 & 14 & 6.2 & 1.29 \\
\hline 40 & 17.5 & 42 & 84 & 28 & 12.4 & 1.24 \\
\hline
\end{tabular}

GPC traces of the two final polymers, PQuiBoc 14 and PQuiBoc 28 , are presented in Figure 2. It can be seen that the polymer peak for PQuiBoc 28 has a small low molar mass shoulder, whereas the PQuiBoc 14 peak appears unimodal. Nevertheless, both polymers were relatively low dispersity $(Ð<1.30)$ and close to the target $D_{\mathrm{p}}$ (taking into account the obtained values are relative to PS standards) with high monomer conversion ( $\geq 84 \%)$. To note, PQuiBoc was soluble in chlorinated organic solvents, such as chloroform, dichloromethane, and nonchlorinated polar aprotic solvents, such as acetone and THF and completely insoluble in cold ether, hexane, toluene, alcohols and water.

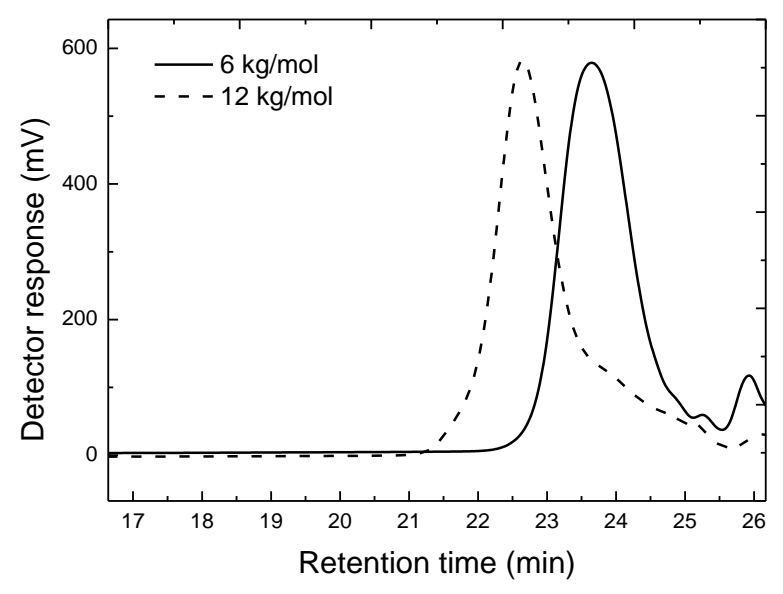


Figure 2. GPC traces of PQuiBoc 14 (6 kg/mol, solid line) and PQuiBoc 28 (12 kg/mol, dotted line) produced by RAFT under following conditions: $[\mathrm{AIBN}]_{0} /[\mathrm{DDMAT}]_{0}=1.0$, QuiBoc:THF $=1: 10$ at $60{ }^{\circ} \mathrm{C}$.

Deprotection of PQuiBoc to produce PQuiOH (Scheme 2) was efficiently and rapidly performed by stirring the polymer solution in DCM using piperidine as a strong base. ${ }^{53}$ The product of the reaction was confirmed by ${ }^{1} \mathrm{H}$ NMR and FTIR spectroscopies where the corresponding peak attributed to the protons of the Boc group (Figure 3a) in ${ }^{1} \mathrm{H}$ NMR and the carbonyl peak at $1760 \mathrm{~cm}^{-1}$ in FTIR disappear (Figure 3b). Moreover, in agreement with previous reports, ${ }^{54}$ the thermal deprotection of PQuiBoc is also possible by heating at $160{ }^{\circ} \mathrm{C}$, as determined by differential scanning calorimetry (DSC, see ESI, Figure S2).

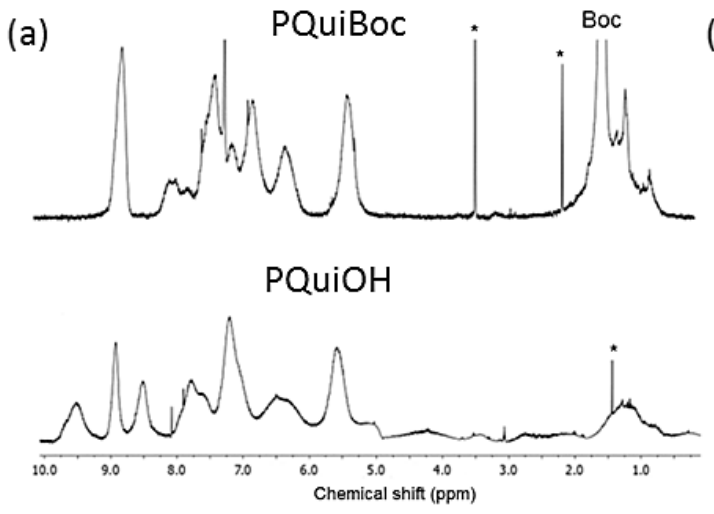

(b)

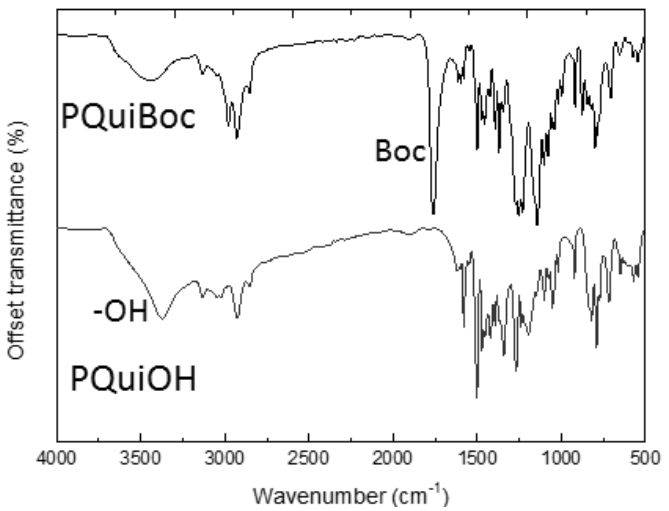

Figure 3. (a) ${ }^{1} \mathrm{H}$ NMR spectra of PQuiBoc and PQuiOH; residual solvent peaks are labelled with asterisks. (b) FTIR spectra of PQuiBoc and PQuiOH, where 'Boc' denotes the carbonyl stretching vibrations of the Boc protecting group.

\section{Surface zinc binding capacity}

To demonstrate the ability of PQuiBoc bind metal oxides, its surface zinc binding capacity, estimated by SEM/EDXA, was selected as an exemplar. As has been shown in previous reports, SEM/EDXA is a semi-quantitative technique that, among other analyses, can allow one to estimate the loading of inorganic nanoparticles on a polymer surface. ${ }^{51}$ To this end, polymer films of PQuiBoc and PS (adopted as a control) were deposited from $5 \mathrm{mg} / \mathrm{ml}$ THF solutions directly onto aluminium SEM substrates. Subsequently, zinc oxide nanoparticles $(\mathrm{ZnO})$ were 
deposited onto the polymer films from a methanol suspension, and, after annealing, the films were thoroughly washed with methanol to remove all non-bound $\mathrm{ZnO}$ from the surface. As previously shown for other polymers, ${ }^{51}$ the molar mass has no effect on the zinc binding capacity of the polymer films, therefore for PQuiBoc, only one molar mass was studied, $12.4 \mathrm{~kg} / \mathrm{mol}\left(D_{\mathrm{p}}\right.$ of 28$)$. It is important to note that the polymer films were annealed at $140{ }^{\circ} \mathrm{C}$ $\left(T_{\mathrm{g}}\right)$ and at $160{ }^{\circ} \mathrm{C}\left(T_{\mathrm{dep}}\right)$, as determined by DSC (see ESI Figure $\mathrm{S} 2$ ).

When PQuiBoc 28 was annealed at $T_{\mathrm{g}}\left(140{ }^{\circ} \mathrm{C}\right), \mathrm{ZnO}$ surface coverage was shown to be $72 \%$ $( \pm 3.1 \%)$, comparable to the polymer annealed at its deprotection temperature $\left(160{ }^{\circ} \mathrm{C}\right)$, i.e. $\mathrm{PQuiOH}_{28}$, for the same time $(69 \%, \pm 2.5 \%)$. These findings indicate that the protected PQuiBoc $_{28}$ is also capable of strongly binding to $\mathrm{ZnO}$, based on hydrogen bonds and dipoledipole interactions. Indeed, the Boc group has two oxygen centres to form hydrogen bonds with the surface hydroxyl groups, present in $\mathrm{ZnO}$ nanoparticles. PS, used as a control, showed negligible $\mathrm{ZnO}$ binding of $4.1 \%( \pm 1.2 \%)$.

These results show that pristine PQuiBoc polymer has several advantages over its deprotected version, PQuiOH: (i) PQuiBoc has a marginally higher ZnO-binding capacity; (ii) PQuiBoc is soluble in common organic solvents typically used in the electrospinning of PS fibres, such as chloroform, tetrahydrofuran and toluene, whereas PQuiOH is not; and (iii) PQuiBoc does not require an extra processing step (chemical or thermal treatment). Thus, PQuiBoc, in its pristine, protected form, was used in a blend with PS to obtain microfibres.

\section{Electrospun polymer fibres}

Since PQuiBoc has demonstrated a high affinity for $\mathrm{ZnO}$ nanoparticles, the amount of PQuiBoc in the blend with PS was kept as low as $20 \%$ (2:8 PQuiBoc:PS mass ratio) to ensure a low production cost, while achieving good loadings of $\mathrm{ZnO}$ on the fibres. It is noteworthy that since polymer molar mass has a pronounced effect on the spinnability of polymers, only PQuiBoc14 $(\sim 6 \mathrm{~kg} / \mathrm{mol})$ has been used in the electrospinning studies since its molar mass more closely 
matches that of the PS employed ( $\sim 5 \mathrm{~kg} / \mathrm{mol})$ in this work. This also demonstrates that such low molar mass polymer blends can be electrospun under these conditions.

Based on previous reports on the electrospinning of PS blends, ${ }^{3,55}$ and good solubility of both PS and PQuiBoc polymers even at high concentrations, the fibers were produced from chloroform (33 wt\% solution). Of note, more dilute solutions ( $<33 \mathrm{wt} \%)$ resulted in inhomogenous fibre formation with beaded morphologies owing to insufficient entanglements between chains. ${ }^{56}$ The electrospinning of PQuiBoc ${ }_{14} / \mathrm{PS}$ mixtures and neat PS (as described in the Experimental section) yielded microfibres (2-6 $\mu \mathrm{m}$ diameter) with a smooth homogenous surface without pores or folds (Figure 4), in line with previous reports. ${ }^{6,8}$

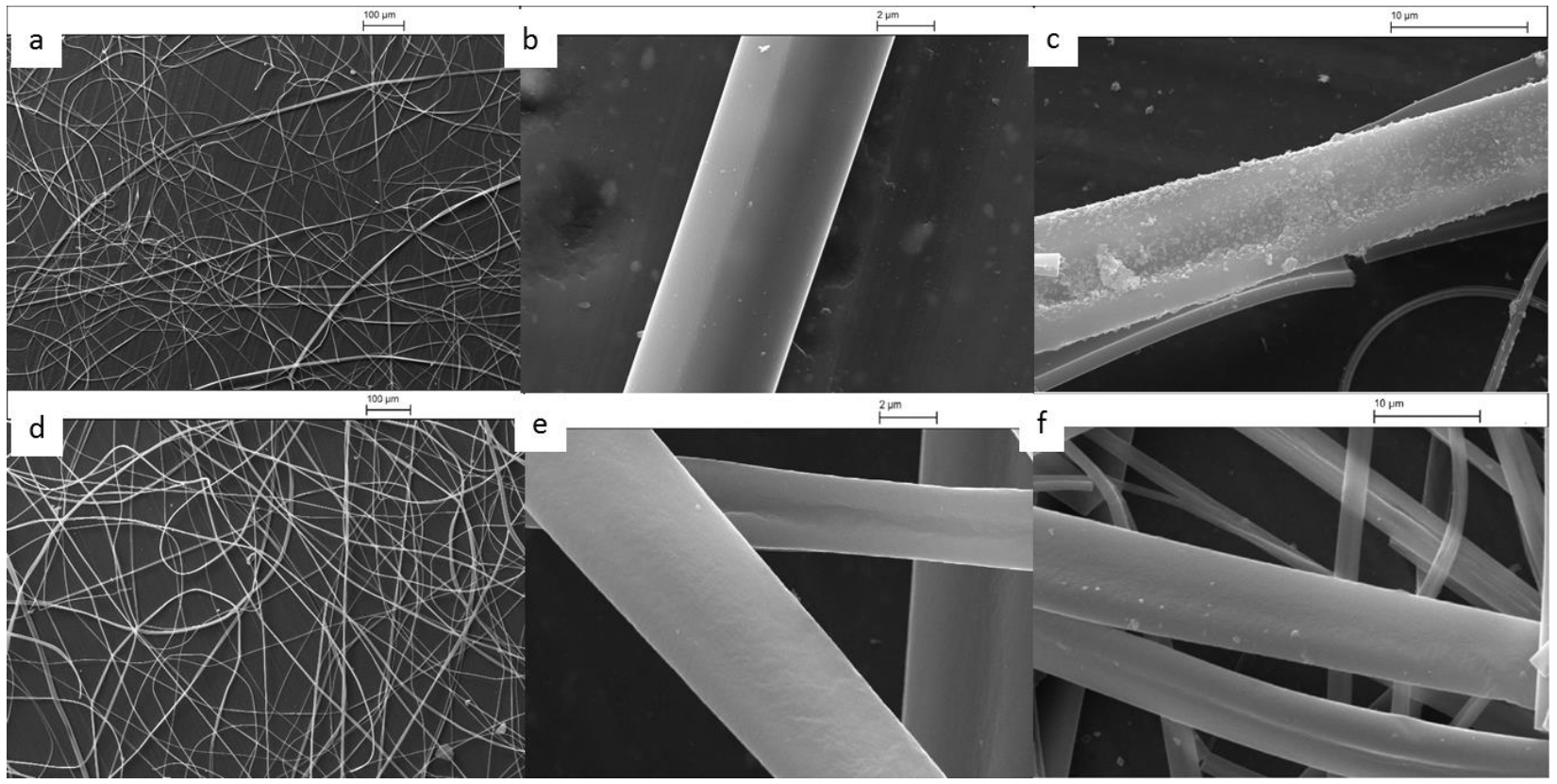

Figure 4. SEM images of pristine electrospun PQuiBoc ${ }_{14} / \mathrm{PS}$ ( $\mathrm{a}$ and $\mathrm{b}$ ) and neat PS (d and e) and after dipping into a suspension of $\mathrm{ZnO}$ nanoparticles and washing with methanol so that only surface-bound $\mathrm{ZnO}$ nanoparticles remain: PQuiBoc $14 / \mathrm{PS} / \mathrm{ZnO}$ (c) and PS/ZnO (f).

The composition of resulting fibres has been revealed by FTIR (Figure 5), where the PQuiBoc $_{14} / \mathrm{PS}$ fibres exhibit a carbonyl band at $1760 \mathrm{~cm}^{-1}$ attributed to the BOC group of the PQuiBoc $_{14}$ material. The two bands at 1226 and $1254 \mathrm{~cm}^{-1}$ are assigned to the $\mathrm{C}-\mathrm{H}$ in-plane bending vibrations of the quinoline and triazole ring protons,${ }^{57}$ whereas the band at $1140 \mathrm{~cm}^{-1}$ arises from the $\mathrm{C}-\mathrm{O}$ bending in the ester group attached to the quinoline system. ${ }^{58}$ Importantly, 
even such low loadings of PQuiBoc 14 (20\% of the total polymer loading) resulted in an increase in hydrophobicity of the fibres, reflected in an increase in the water contact angle from $137^{\circ}$ to $152^{\circ}$ (Figure 5), despite the presence of the oxygen and nitrogen atoms.

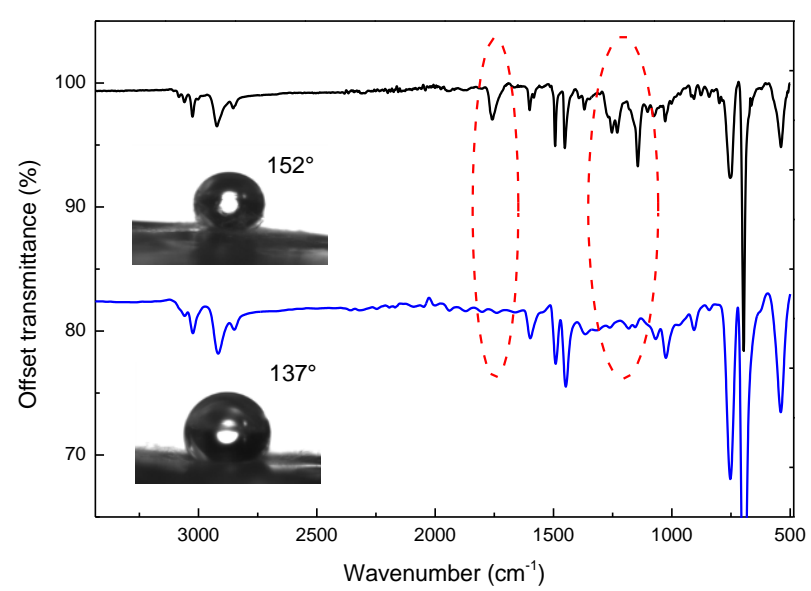

Figure 5. FTIR spectra of PQuiBoc 14 /PS microfibres (black) and PS microfibres (blue) electrospun from $33 \mathrm{wt} \%$ chloroform solution at $20 \mathrm{kV}, 3.5 \mathrm{ml} / \mathrm{h}$ feed rate and $20 \mathrm{~cm}$ needleto-collector distance.

To load the fibres with $\mathrm{ZnO}$ nanoparticles, the fibre mats were dipped into a $\mathrm{ZnO}$ suspension in methanol for $10 \mathrm{~min}$ and then washed multiple times with methanol and sonicated in methanol to remove any $\mathrm{ZnO}$ that was not strongly bound to the fibres. The uptake of $\mathrm{ZnO}$ nanoparticles was estimated by SEM/EDXA to be $29 \%( \pm 0.4 \%)$ by PQuiBoc ${ }_{14} / \mathrm{PS}$ microfibres and only $11 \%( \pm 2.7 \%)$ by neat PS microfibres (Figure 4, c and f). The presence of tightly bound $\mathrm{ZnO}$ on the surface of microfibres is accompanied by a decrease of the contact angle to $92^{\circ}$, reflecting the more hydrophilic nature of the $\mathrm{ZnO}$ particles.

Thus, the combination of PS used as a matrix and PQuiBoc used as functional metal-binding component is employed in fabrication of composite microfibres with increased surface area and high zinc-binding capacity. The introduction of only $20 \%$ PQuiBoc into the blend results in the fabrication of smooth microfibres with an almost three-fold increase in surface $\mathrm{ZnO}$ loading, as compared to neat PS fibres, critical for applications such as photocatalysis, antibacterial wound dressings and UV-absorbing fabrics. Of specific note, using PQuiBoc 
instead of PQuiOH provides several advantages: (i) allows electrospinning from the same single solvent (chloroform); (ii) decreases the number of processing steps and thus the fabrication costs of the fibres, whilst (iii) providing comparable (if not marginally higher) zincbinding capacity than PQuiOH. Furthermore, the high affinity of PQuiBoc for metals gives potential for this approach to be used in heavy metal sequestration in waste water treatment or as absorbents for sample preparation in atomic spectroscopy.

\section{Conclusions}

This paper reports the design and synthesis of a new styrene-based monomer, intended to incorporate the zinc-binding moiety of 8-hydroxyquinoline, and its subsequent polymerisation by RAFT. RAFT polymerisation herein yielded well-defined polymers, PQuiBoc, with relative degrees of polymerisation of 14 and 28 (against PS standards), furnished with carboxylic acid terminal functionality with the potential of copolymerisation or derivatisation. Suitable conditions for RAFT were defined as a 1:1 ratio of initiator to CTA $\left([\mathrm{AIBN}]_{0} /[\mathrm{DDMAT}]_{0}=\right.$ 1.0) at a relatively high dilution of 1:10 monomer:THF at $60{ }^{\circ} \mathrm{C}$. As shown by SEM/EDXA, the $\mathrm{ZnO}$ binding capacity of protected PQuiBoc was comparable to (if not marginally higher than) deprotected PQuiOH, which allowed the fabrication of electrospun fibres from a PQuiBoc/PS blend without the need for deprotection (removal of the Boc group). Electrospun PQuiBoc/PS microfibres with only $20 \%$ content of PQuiBoc had almost three-fold higher loadings of $\mathrm{ZnO}(29 \%)$ in comparison to neat PS microfibres $(11 \%)$. Thus, the new zincbinding PQuiBoc material allows straightforward and relatively inexpensive fabrication of composite polymeric fibres which provide a high surface loading of $\mathrm{ZnO}$ nanoparticles for future use in photocatalysis or wound dressings with a high surface area, suitable for membranes in heavy metal sequestration.

\section{Acknowledgements}


The research leading to these results has received funding from the European Union Seventh Framework Programme (FP7/2011 under grant agreement ESTABLIS no. 290022). A.I. thanks Dr Roger Hiorns and Dr Melanie Pedeutour (University of Pau).

\section{References}

1 F. L. Matthews and R. D. Rawlings, Composite Materials: Engineering and Science, Elsevier, 1999.

2 A. Greiner and J. H. Wendorff, Angew. Chem. Int. Ed. Engl., 2007, 46, 5670-703.

3 Z.-M. Huang, Y.-Z. Zhang, M. Kotaki and S. Ramakrishna, Compos. Sci. Technol., 2003, 63, 2223-2253.

4 S. Huan, G. Liu, G. Han, W. Cheng, Z. Fu, Q. Wu and Q. Wang, Materials, 2015, 8, 2718-2734.

5 Y. Hada, H. Shikuma, H. Ito and T. Kikutani, Fibers Polym., 2005, 6, 19-27.

6 J. Zheng, A. He, J. Li, J. Xu and C. C. Han, Polymer, 2006, 47, 7095-7102.

7 G.-T. Kim, J.-S. Lee, J.-H. Shin, Y.-C. Ahn, Y.-J. Hwang, H.-S. Shin, J.-K. Lee and C.-M. Sung, Korean J. Chem. Eng., 2005, 22, 783-788.

8 J. Lin, B. Ding and J. Yu, ACS Appl. Mater. Interfaces, 2010, 2, 521-8.

9 J. Lin, B. Ding, J. Yang, J. Yu and G. Sun, Nanoscale, 2012, 4, 176-82.

10 C.-C. Kuo, Y.-C. Tung and W.-C. Chen, Macromol. Rapid Commun., 2010, 31, 65-70.

11 H. Zhu, S. Qiu, W. Jiang, D. Wu and C. Zhang, Environ. Sci. Technol., 2011, 45, 4527-31.

12 J. Lin, Y. Shang, B. Ding, J. Yang, J. Yu and S. S. Al-Deyab, Mar. Pollut. Bull., 2012, 64, 347-52.

13 C. Shin, G. G. Chase and D. H. Reneker, Colloids Surfaces A Physicochem. Eng. Asp., 2005, 262, 211-215.

14 M. W. Lee, S. An, S. S. Latthe, C. Lee, S. Hong and S. S. Yoon, ACS Appl. Mater. Interfaces, 2013, 5, 10597-604.

15 H. An, C. Shin and G. G. Chase, J. Memb. Sci., 2006, 283, 84-87.

16 Z. Liu, J. Yan, Y.-E. Miao, Y. Huang and T. Liu, Compos. Part B Eng., 2015, 79, $217-$ 223.

17 S. C. Baker, N. Atkin, P. A. Gunning, N. Granville, K. Wilson, D. Wilson and J. Southgate, Biomaterials, 2006, 27, 3136-3146. 
18 T. Ruotsalainen, J. Turku, P. Hiekkataipale, U. Vainio, R. Serimaa, G. ten Brinke, A. Harlin, J. Ruokolainen and O. Ikkala, Soft Matter, 2007, 3, 978.

19 M. Ma, V. Krikorian, J. H. Yu, E. L. Thomas and G. C. Rutledge, Nano Lett., 2006, 6, 2969-2972.

20 M. Ma, R. M. Hill, J. L. Lowery, S. V Fridrikh and G. C. Rutledge, Langmuir, 2005, 21, 5549-54.

21 G. Liu, J. Ding, L. Qiao, A. Guo, B. P. Dymov, J. T. Gleeson, T. Hashimoto and K. Saijo, Chem. - A Eur. J., 1999, 5, 2740-2749.

22 K. Müller, J. F. Quinn, A. P. R. Johnston, M. Becker, A. Greiner and F. Caruso, Chem. Mater., 2006, 18, 2397-2403.

23 O. J. Rojas, G. A. Montero and Y. Habibi, J. Appl. Polym. Sci., 2009, 113, 927-935.

24 K. C. M. Nair and S. Thomas, Polym. Compos., 2003, 24, 332-343.

25 R. Sen, B. Zhao, D. Perea, M. E. Itkis, H. Hu, J. Love, E. Bekyarova and R. C. Haddon, Nano Lett., 2004, 4, 459-464.

26 J. K. Kim and H. Ahn, Macromol. Res., 2008, 16, 163-168.

27 B. Ding, J. Lin, X. Wang, J. Yu, J. Yang and Y. Cai, Soft Matter, 2011, 7, 8376.

28 M. Kobayashi, M. Egashira and T. Konno, Mater. Sci. Forum, 2007, 561-565, $663-$ 666.

29 Z. Jiang, L. D. Tijing, A. Amarjargal, C. H. Park, K.-J. An, H. K. Shon and C. S. Kim, Compos. Part B Eng., 2015, 77, 311-318.

30 B. Pule, S. Degni and N. Torto, Water SA, 2014, 41, 27.

31 H. F. Moafi, A. F. Shojaie and M. A. Zanjanchi, Thin Solid Films, 2011, 519, 36413646.

32 S. Baruah, C. Thanachayanont and J. Dutta, Sci. Technol. Adv. Mater., 2008, 9, 025009.

33 Y. Hamada, T. Sano, M. Fujita, T. Fujii, Y. Nishio and K. Shibata, Jpn. J. Appl. Phys., 1993, 32, L514-L515.

34 R. Wang, Y. Cao, D. Jia, L. Liu and F. Li, Opt. Mater., 2013, 36, 232-237.

35 N. Du, Q. Mei and M. Lu, Synth. Met., 2005, 149, 193-197.

36 Z. Jin and J. Wang, J. Mater. Chem. C, 2014, 2, 1966.

37 V. Prachayasittikul, S. Prachayasittikul, S. Ruchirawat and V. Prachayasittikul, Drug Des. Devel. Ther., 2013, 7, 1157-78.

38 J. T. Lai, D. Filla and R. Shea, Macromolecules, 2002, 35, 6754-6756. 
39 S.-X. Li, L.-R. Feng, X.-J. Guo and Q. Zhang, J. Mater. Chem. C, 2014, 2, 3517.

40 D. T. W. Toolan, N. Pullan, M. J. Harvey, P. D. Topham and J. R. Howse, $A d v$. Mater., 2013, 25, 7033-7.

41 D. Gelman and S. L. Buchwald, Angew. Chemie-International Ed., 2003, 42, 59935996.

42 G. Moad, M. Chen, M. Haeussler, A. Postma, E. Rizzardo and S. H. Thang, Polym. Chem., 2011, 2, 492-519.

43 G. Moad, E. Rizzardo and S. H. Thang, Polymer, 2008, 49, 1079-1131.

44 G. Gody, T. Maschmeyer, P. B. Zetterlund and S. Perrier, Nat. Commun., 2013, 4, 2505.

45 J. Kolomanska, P. Johnston, A. Gregori, I. Fraga Domínguez, H.-J. Egelhaaf, S. Perrier, A. Rivaton, C. Dagron-Lartigau and P. D. Topham, RSC Adv., 2015, 5, 6655466562.

46 I. F. Domínguez, J. Kolomanska, P. Johnston, A. Rivaton and P. D. Topham, Polym. Int., 2015, 621-630.

47 H. Erothu, A. Sohdi, A. C. Kumar, A. J. Sutherland, C. Dagron-Lartigau, A. Allal, R.

C. Hiorns and P. D. Topham, Polym. Chem., 2013, 4, 3652.

48 S. Kulkarni, C. Schilli, B. Grin, A. H. E. Mueller, A. S. Hoffman and P. S. Stayton, Biomacromolecules, 2006, 7, 2736-2741.

49 D. H. Nguyen and P. Vana, Polym. Adv. Technol., 2006, 17, 625-633.

50 C. Li, J. Han, C. Y. Ryu and B. C. Benicewicz, Macromolecules, 2006, 39, 31753183.

51 A. Isakova, P. D. Topham and A. J. Sutherland, Macromolecules, 2014, 47, 25612568.

52 S. I. Cauet and K. L. Wooley, J. Polym. Sci. Part A-Polymer Chem., 2010, 48, $2517-$ 2524.

53 D. K. Mohapatra and K. A. Durugkar, Arkivoc, 2005, 20-28.

54 K. E. Krakowiak and J. S. Bradshaw, Synth. Commun., 1996, 26, 3999-4004.

55 A. E. Senador, M. T. Shaw and P. T. Mather, MRS Proc., 2011, 661, KK5.9.

56 L. Wang, P. D. Topham, O. O. Mykhaylyk, H. Yu, A. J. Ryan, J. P. A. Fairclough and W. Bras, Macromol. Rapid Commun., 2015, 36, 1437-1443.

57 N. Puviarasan, V. Arjunan and S. Mohan, Turkish J. Chem., 2004, 28, 53 - 65.

58 X. Zhang, S. Shi, Q. Liu, J. Zhou, J. Ye and C. Yu, Chem. Commun., 2011, 47, 6359. 

For Table of Contents use only

Design, synthesis and RAFT polymerisation of a quinoline-based monomer for use in electrospun composite microfibres

Anna Isakova, ${ }^{a}$ Olga Efremova, ${ }^{b}$ Nikki Pullan, ${ }^{a}$ Larry Lüer, ${ }^{c}$ Paul D. Topham ${ }^{a *}$

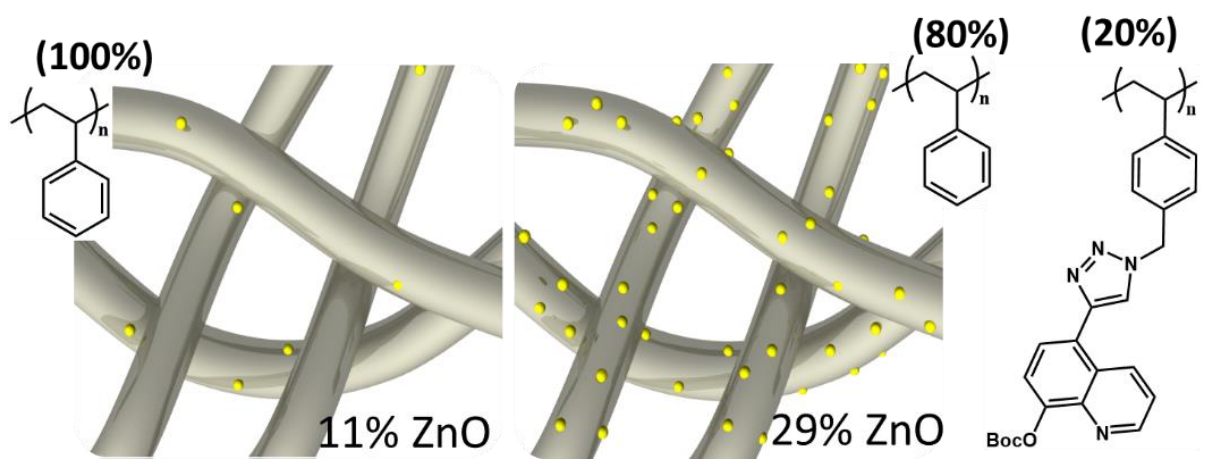

